\title{
Possibility of tuning shunt circuits for multimodal damping of vibrations of structure with piezoelectric element
}

\author{
Dmitrii A. Oshmarin, Maksim A. Iurlov, Nataliya V. Sevodina, Nataliia A. Iurlova \\ Institute of Continuous Media Mechanics of the Ural Branch of RAS, Perm, Russia \\ oshmarin@icmm.ru, bttp:/ /orcid.org/0000-0002-9898-4823 \\ yurlovm@icmm.ru,bttp://orcid.org/0000-0003-1507-6617 \\ natsev@icmm.ru,bttp:/ / orcid.org/0000-0001-9374-7135 \\ yurlova@icmm.ru, bttp:/ /orcid.org/0000-0003-3497-0358
}

\begin{abstract}
For the selecting of the parameters of the shunt circuit that provide multimodal damping of vibrations for the corresponding piezoelectric element location an approach is proposed. This technique is based on a solution to the natural vibration problem for an electroelastic structure with an attached piezoelectric element that is shunted with a single external resonant electric circuit. The solution to the problem is complex natural vibration frequencies. The analysis of their behaviour in the space of external circuit parameters (resistance - inductance) allows one to reveal the possibility of natural vibrations control at several frequencies (multimodal vibration damping). The applicability of the proposed approach is demonstrated using a shell structure in the form of a semi-cylinder with a piezoelectric element attached to its surface and shunted with a singlebranched resonant electric circuit. Some options are considered that provide multimodal damping at several separated frequencies, which are not from the complete frequency range, and at all the frequencies, which are included in a specified frequency range.
\end{abstract}

KEYWORDS. Electroelasticity; External electric circuit; Piezoelectric element; Natural vibrations; Numerical modeling; Multimodal damping.

\section{OPEN ACCESS}

Citation: Oshmarin, D., Sevodina, N., Iurlov, M., Iurlova, N., Possibility of tuning shunt circuits for multimodal damping of vibrations of structure with piezoelectric element, Frattura ed Integrità Strutturale, 49 (2019) 800-813.

Received: 28.03.2019

Accepted: 16.05.2019

Published: 01.07.2019

Copyright: (C) 2019 This is an open access article under the terms of the CC-BY 4.0, which permits unrestricted use, distribution, and reproduction in any medium, provided the original author and source are credited.

\section{INTRODUCTION}

$\mathrm{T}$ he idea of using piezoelectric elements with connected external electric circuits to damp the vibrations of mechanical structures has proved highly efficient and attractive, especially for engineering applications with strict size (miniaturization) and weight (elements of space and aircraft facilities) limits. Piezoelectric elements connected to external circuits (shunted peizoelectric elements) attached to the mechanical structure form energy dissipation devices, which provide additional damping of vibrations. Due to the piezoelectric effect, part of the mechanical vibration energy is transformed into electrical energy, which can be dissipated through the shunt circuit as heat or electromagnetic radiation, 
thereby generating the mechanism of passive control of vibration. Because peizoelectric elements and components of the external circuits are small in weight, such a system does not lead to serious changes in the spectral pattern of the initial structure and can be used as a complementary device to developed technical solutions. The possibility of applying such devices to damping the vibrations of a mechanical structure was first demonstrated by Forward [1] and theoretically substantiated by Hagood and von Flotow [2].

A key to the problem of vibration damping with shunted piezoelectric elements is to find the parameters of a simplest shunt that is most effective in damping the vibrations of a particular structure at a group of frequencies in the specified frequency range. The shunt circuit should meet the following requirements: it should provide maximum damping of mechanical vibrations and preserve its stability; it should operate without a power source; its design costs and weight should be reduced to a minimum; and, since the circuit is integrated in the structure, the size of its elements should be minimal [3].

Over the last decade, a variety of passive shunt circuits have been designed and rated. According to the classification presented in Moheimani and Fleming [3], passive shunts can be linear or nonlinear. Among the linear passive circuits, one can distinguish between the resistive (including only resistors) and resonant (including resistors and inductors) circuits.

The resistive circuits used to shunt piezoelectric elements cause the structure to behave as if it is made of viscoelastic material. The use of resonant $R L$ circuits consisting of a parallel- or series-connected inductance coil and resistor leads to the creation of the electric oscillatory circuit due to the interaction of the external circuit inductance and inherent capacitance of the piezoelectric element. As a result, the frequency spectrum of the system (the structure with a piezoelectric element and external circuit) extends to include an additional resonance frequency (eigenfrequency of the oscillatory circuit).

By varying the parameters of the elements of the electric circuit, one can adjust this additional resonance to the natural vibration frequency of the structure. This allows the energy of vibrations to be transferred to the external circuit, which provides an effective suppression of the structure vibrations at a given frequency. In practice, the requirements commonly imposed on structures are the absence of vibrations at the frequencies of a certain group in some excitation frequency range. Therefore, the development of techniques for multimodal damping of structure vibrations is an urgent problem. At present, one can distinguish the following basic approaches to multimodal damping:

- the employment of one piezoelectric element and a complex external circuit in which the possibility of damping several modes of vibrations is realized in one way or another [4-15];

- the usage of several piezoelectric elements forming an integrated network and connected by one or another method to a single external circuit [16-22];

- the usage of several piezoelectric elements, each having its own electrical circuit that is not coupled with others to form a joint circuit [23-27].

Each of these approaches has its own advantages and drawbacks. Thus, for example, in the presence of complicated branched circuits the complexity of their tuning increases, because each branch of the shunt circuit cannot be considered as a separate independent shunt [13]. In this case, it is advisable to use blocking or conducting circuits connected in series to shunt branches [9, 12], which allows one to consider them as independent. However, in practice this leads to very cumbersome electrical circuits due to the use of many inductive elements, which certainly affects the mass characteristics of damping devices. This problem can be partially solved by using electronic analogues of inductive elements. However, such a substitution is only an approximation to ideal inductive elements and does not completely eliminate the necessity of tuning the branches and determining the circuit parameters [7].

The difficulty with the circuit tuning can be readily solved with the use of several piezoelectric elements connected to simple RL circuits, since in this case each piezoelectric element with a connected circuit represents an isolated system and parameters of the shunt circuits can be selected independently. However, as shown in [4], in the case of multimodal damping, this approach is not necessarily successful because a great number of elements in the circuit can essentially increase the mass of the structure and have an ill effect on its dynamic characteristics.

The variety of approaches and techniques based on the application of piezoelectric elements connected to external electric circuits for the problem of multimodal vibration damping was demonstrated in the paper [28]. But at the same time the authors proposed new technique for solving the stated problem. The essence of their technique is in consideration of an impedance of a shunting circuit as a controller in the state space of the model of electromechanical system. In order to achieve multimodal vibration damping it is required to find the electric circuit that has the desirable impedance.

Hence, nowadays, the problem accosiated with the use of piezoelectric elements to damp several vibration modes still requires further investigation and development of new approaches to the implementation of multimodal damping.

This paper presents one possible application of the natural vibration problem for electroelastic structures that include piezoelectric elements connected to external circuits. The results of the solution to the problem are complex values of 
natural vibration frequencies where the real part defines the circular natural vibration frequency of the structure and the imaginary part defines its vibration damping rate (i.e. appears to be the parameter of structural vibration damping). A previous analysis was performed of the behaviour of imaginary parts of complex eigenfrequencies in the space of external circuit parameters (resistance - inductance) while tuning the external circuit to a given frequency of vibrations of the structure. This analysis revealed some peculiarities of the behaviour of damping ratios at certain frequencies, which also allowed for a sufficiently high damping rate at some other frequencies. From this, it may be possible to use one single piezoelectric element and one single resonant $R L$ circuit to damp vibrations at several frequencies with an appropriate choice of parameters. This work is devoted to the demonstration of such a possibility.

\section{MATHEMATICAL STATEMENT OF THE PROBLEM}

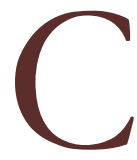

onsider a piecewise homogeneous body of volume $V=V_{1}+V_{2}$, where volume $V_{1}$ is composed of homogeneous elastic parts and volume $V_{2}$ of piezoelectric elements. Part of the surface of volume $V_{2}$ is covered with electrodes. To one of the electrode surfaces of volume $V_{2}$ is attached an external passive electrical circuit of arbitrary configuration, consisting of resistive (resistance $R$ ), inductive (inductance $L$ ) and capacitive (capacitance C) elements.

One of the quite efficient problems in mechanics that is used for optimization of dissipative properties of deformable solids is problem on natural vibrations of bodies in which energy dissipation occurs during vibrations. Mathematical statement of this problem and its applications for studying natural vibrations of viscoelastic bodies were proposed in [29]. Algorithm of numerical implementation of the problem with the aid of capabilities of the ANSYS software package was presented in [30]. The approach proposed in these papers has been applied for investigating natural vibrations of electroelastic bodies with external electric circuits.

The variational equation for natural vibrations of the body consisting of elastic and piezoelectric elements is constructed based on the relations of linear theory of elasticity and quasi-static Maxwell equations [31-34]:

$$
\begin{aligned}
& \int_{V_{1}}\left(\sigma_{i j} \delta \varepsilon_{i j}+\rho \ddot{u}_{i} \delta u_{i}\right) d V+\int_{V_{2}}\left(\sigma_{i j} \delta \varepsilon_{i j}-D_{i} \delta E_{i}+\rho \ddot{u}_{i} \delta u_{i}\right) d V+ \\
& +\sum_{p=1}^{n_{L}} \frac{1}{L_{p}} \iint\left(\varphi_{1}^{L_{p}}-\varphi_{2}^{L_{p}}\right) \delta \varphi d t d t+\sum_{q=1}^{n_{R}} \frac{1}{R_{q}} \int\left(\varphi_{1}^{R_{q}}-\varphi_{2}^{R_{q}}\right) \delta \varphi d t+\sum_{r=1}^{n_{C}} C_{r}\left(\varphi_{1}^{C_{r}}-\varphi_{2}^{C_{r}}\right) \delta \varphi=0
\end{aligned}
$$

Here $D_{i}, E_{i}$ are the components of electric flux density and electric field intensity vectors; $\sigma_{i j}$ are the components of the symmetric Cauchy stress tensor, $\varepsilon_{i j}$ are the components of the linear strain tensor, $u_{i}$ are the components of the displacement vector, $\varphi$ is electric potential, $\varphi_{1}-\varphi_{2}$ is the potential difference on external circuit element, $n_{L}, n_{R}, n_{C}$ are the numbers of inductive, resistive and capacitive elements respectively, $L_{p}, R_{q}, C_{r}$ are inductance, resistance and capacitance values for corresponding circuit element.

For the electric field, the potentiality condition is fulfilled:

$$
\varphi_{, i}=-E_{i}
$$

For elastic parts $\left(V_{1}\right)$, the following physical equations of the linear theory of elasticity hold true:

$$
\sigma_{i j}=C_{i j k l} \varepsilon_{k l}
$$

For isothermal processes, the electroelastic parts $\left(V_{2}\right)$ obey the following physical relations: 


$$
\left.\begin{array}{c}
\sigma_{i j}=\bar{C}_{i j k l} \varepsilon_{k l}-\beta_{i j k} E_{k} \\
D_{k}=\beta_{i j k} \varepsilon_{i j}+e_{k i} E_{i}
\end{array}\right\}
$$

where $C_{i j k l}$ and $\bar{C}_{i j k l}$ are the components of the tensors of elastic constants, $\beta_{i j k}$ and $e_{k i}$ are the components of the tensors of piezoelectric and dielectric coefficients.

For the examined system, the restrictions are set in the form of mechanical and electrical boundary conditions. The mechanical boundary conditions are given as:

$$
S_{\sigma}: \quad \sigma_{i j} n_{j}=0, \quad S_{u}: u_{i}=0
$$

where $S=S_{\sigma}+S_{u}$ is the surface of full volume $V$.

The expressions for the electrical boundary condition on the piezoelectric parts confined in volume $V_{2}$ read as follows:

$$
\left.\varphi\right|_{\Omega_{\ell l}^{\varphi}}=0
$$

where $\Omega_{e l}^{\varphi}$ is the part of the electrode surface of the volume $V_{2}$, with the prescribed electric potential. In the absence of the electrode covering all electrical boundary conditions take zero values.

The eigenvectors of vibrations at zero-valued boundary conditions (5-6) are sought in the following form:

$$
u_{i}(x, t)=\bar{u}_{i}(\vec{x}) e^{i \omega t}, \quad \varphi(x, t)=\bar{\varphi}(\vec{x}) e^{i \omega t}
$$

where $\omega=\omega_{\mathrm{Re}}+i \omega_{\mathrm{Im}}$ is the complex eigenfrequency, in which $\omega_{\mathrm{Re}}$ corresponds to the vibration eigenfrequency itself, $\omega_{\operatorname{Im}}$ corresponds to the decay rate of vibration (damping index) and $\bar{u}_{i}(\vec{x}), \bar{\varphi}(\vec{x})$ are eigenmodes of vibrations.

A complete mathematical formulation of the problem is given in [33-34]. The stated problem is solved by the finite element method, using the algorithm elaborated by the authors of this paper using ANSYS package possibilities and program written in FORTRAN language.

As shown in [2], the series resonant electric circuit, shunting the piezoelectric element can decrease oscillations for only one selected frequency, to which the external circuit is tuned by choosing the appropriate parameters $R$ and $L$.

Here will be given explanations related to the process of tuning. A piezoelectric element, by virtue of its nature, has capacitive properties and in the presence of the external $R L$-circuit forms a series resonance oscillatory $R L C$-circuit. This leads to the appearance of additional eigenfrequency $\omega^{e}$ in the natural frequencies spectrum of the base structure, which is due to the interaction of the inductive element and the inherent capacitance of the piezoelectric element. As a result, the spectrum of the eigenfrequencies of the structure with a piezoelectric element and an external circuit is formed. This additional eigenfrequency $\omega^{e}$ can vary over a wide range due to changing the parameters of the external circuit. During the process of tuning this additional frequency is brought closer and closer to the eigenfrequency of the structure $\omega^{n}$ until they coincide.

Since in the complex eigenfrequency (according to the mathematical formulation of the problem) the real part characterizes the frequency of vibrations, one of the conditions for selecting parameters of the shunting circuit can be given as

$$
\omega_{\mathrm{Re}}^{n}=\omega_{\mathrm{Re}}^{e}
$$

From the one hand the performed numerical investigations [35] showed, that in the space of R- $L$ parameters there is a set of points corresponding to different values of resistance and inductance, at which condition (8) is satisfied. From the other hand the performed numerical investigations have revealed that the damping index $\omega_{\text {Im }}^{e}$ related to the additional complex frequency of the electric oscillatory circuit varies within very wide range and has, in the space of electric circuit parameters $R$ and $L$, a great number of local extrema, which appear at the instant of convergence of electric circuit 
damping index with the damping indices $\omega_{\operatorname{Im}}^{n}$ of the complex natural vibration frequencies related to the structure. However for each natural vibration frequency of the structure, there exists only one global extremum of the damping index over the entire space of possible values of the $R-L$ parameters [35].

As a result, the condition providing a unambiguous determination of the optimal values of shunting circuit parameters of $\mathrm{R}_{j}^{o p t}$ and $L_{j}^{o p t}$, at which the highest damping rate of the appropriate vibration mode can be reached, can be was obtained in the form of (9)

$$
\left|\omega_{\operatorname{Im}}^{n}\right| \rightarrow \max
$$

The results obtained during numerical calculations show that in the case of application of a resonant circuit for damping of vibrations at one mode there exist options when damping of vibrations is also observed at some other modes. This means it may be possible to implement a single piezoelectric element shunted with one single resonant $R L$ circuit to damp vibrations on several modes.

Imaginary parts of complex natural frequencies $\omega_{\mathrm{Im}}$ were chosen as a parameter to formulate the criteria for shunt circuit parameters selection in order to damp vibrations on several modes.

Taking into account the complex conjugation of the obtained eigenvalues $\omega=\omega_{\mathrm{Re}} \pm i \omega_{\mathrm{Im}}$, the ones are chosen are those that have a positive imaginary part. This choice provides the damped character of vibration processes under consideration. Representing eigenvectors (7) in trigonometric form (10), allows seeing that exactly the imaginary part of complex natural vibration frequency $\omega_{\mathrm{Im}}$ determines the damping properties of a structure (in this case, the rate of decay of the vibration amplitude)

$$
\{u\}=\left\{u_{0}\right\} e^{-\omega_{\mathrm{Im}} t}\left(\cos \left(\omega_{\mathrm{Re}} t\right)+i \sin \left(\omega_{\mathrm{Re}} t\right)\right)
$$

Analysis of the behaviour of the imaginary parts in the R-L parameters space shows the existence of variants for which damping indices related to different frequencies appeared to be equal, meaning that the damping rates for these frequencies is equal. In this regard, the following condition for selecting the parameters of a series resonant $R L$-circuit was chosen: in order to damp several vibration modes, the parameters of the shunt circuit should correspond to the point in the $R-L$ space where absolute values damping ratios coincide and are maximal.

\section{DEFINITION OF SHUNT CIRCUIT PARAMETERS FOR DAMPING STRUCTURAL VIBRATIONS AT SEVERAL FREQUENCIES}

he applicability of the proposed approach can be demonstrated using a thin-walled shell in the form of a semicylinder that is clamped at the edges and simply supported along the generatrix (Fig. 1).

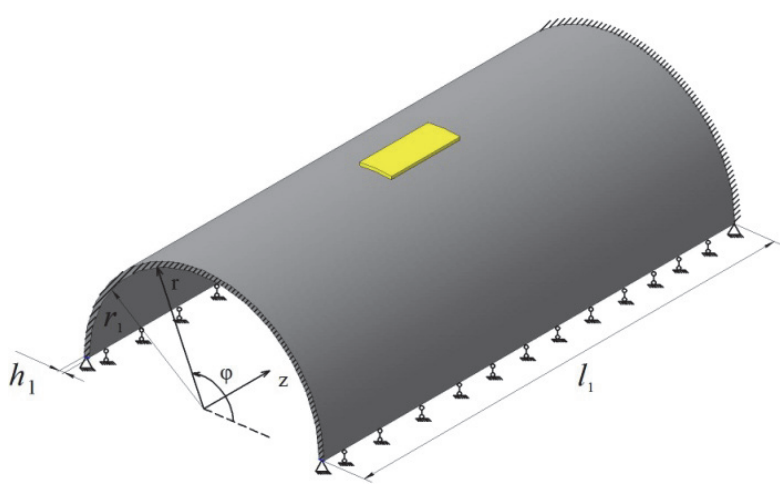

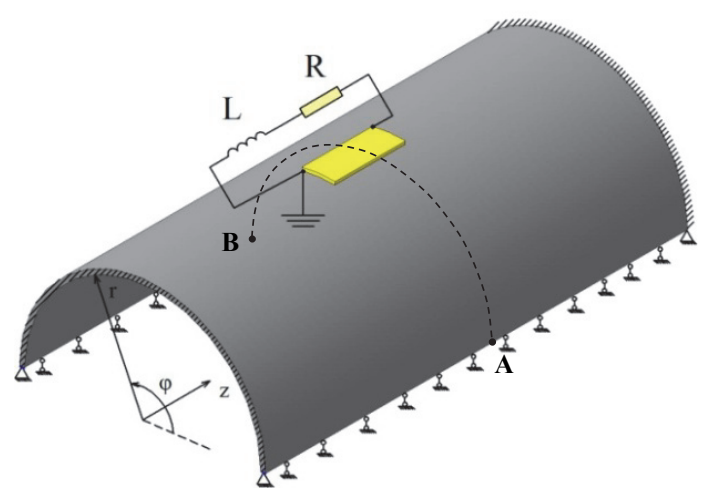

$\mathrm{b}$

Figure 1: The design models of cylindrical shell with attached piezoelectric element (a) and cylindrical shell with attached piezoelectric element connected to series $R L$ circuit (b). 
The geometrical dimensions of the shell are as follows: $r_{1}=76 \mathrm{~mm} \quad l_{1}=300 \mathrm{~mm}, h_{1}=0.25 \mathrm{~mm}$. The shell is made of elastic isotropic material having the following characteristics: $E=1.96 \cdot 10^{11} \mathrm{~Pa}, \quad v=0.3, \quad \rho=7700 \mathrm{~kg} / \mathrm{m}^{3}$. The piezoelectric element, which is attached to the surface of the shell has the shape of the ring segment with the following dimensions: $r_{p}=76.25 \mathrm{~mm}, \varphi_{p}=15.08^{\circ}, h_{p}=0.36 \mathrm{~mm}$. The centre of mass of the piezoelectric element is $15 \mathrm{~mm}$ away from the clamped edges and is shifted by $90^{\circ}$ from the simply supported generatrix along the angular coordinate. The piezoelectric element is made of peizoceramics PZT-4 polarised along the $r$-axis and having the following characteristics (in the cylindrical coordinate system): $C_{11}=C_{22}=13.9 \cdot 10^{10} \mathrm{~Pa}, \quad C_{12}=7.78 \cdot 10^{10} \mathrm{~Pa}, \quad C_{13}=C_{23}=7.43 \cdot 10^{10} \mathrm{~Pa}$, $C_{33}=11.5 \cdot 10^{10} \mathrm{~Pa}, \quad C_{44}=3.06 \cdot 10^{10} \mathrm{~Pa}, \quad C_{55}=C_{66}=2.56 \cdot 10^{10} \mathrm{~Pa}, \quad \beta_{31}=\beta_{32}=-5.2 \mathrm{C} / \mathrm{m}^{2}, \quad \beta_{33}=15.1 \mathrm{C} / \mathrm{m}^{2}$, $\beta_{52}=\beta_{61}=12.7 \mathrm{C} / \mathrm{m}^{2}, e_{11}=e_{22}=6.45 \cdot 10^{-9} \mathrm{~F} / \mathrm{m}, e_{33}=5.62 \cdot 10^{-9} \mathrm{~F} / \mathrm{m}, \rho=7700 \mathrm{~kg} / \mathrm{m}^{3}$. The upper and lower surfaces of the piezoelectric element are covered with electrodes. The external series $R L$ circuit is connected to piezoelectric element electrodes (Fig. 1b).

The solution of the natural vibration problem for the structure with an attached piezoelectric element and without an external circuit shows that in the frequency range $0-1500 \mathrm{~Hz}$ there are 15 natural vibration frequencies $f=2 \pi \omega$.

For the specified piezoelectric element location, the electric potential generated on its electrodes appears to be sufficient for registration and further operation only at five frequencies from the specified frequency range: 1st, 4th, 5th, 12th and 15th (coupled modes of vibration). An almost negligible electric potential at other frequencies indicates that the mode shapes for these frequencies are such that the piezoelectric element either remains undeformed or it deforms symmetrically, which leads to zero-value total electric charge (uncoupled modes of vibration) [36].

On the basis of the solution to the natural vibration problem (1)-(7), values of natural vibration frequencies were obtained for the shell without an external circuit (real) and with a series RL-circuit (complex), with the parameters chosen to reach maximal damping of corresponding frequencies from the specified frequency range. Tab. 1 presents the five frequencies for which generated electric potential is sufficient for the efficient operation of the piezoelectric element (only for coupled modes). Tab. 1 also shows the optimal shunt circuit parameters with corresponding complex eigenfrequency values to damp vibrations at these frequencies separately.

\begin{tabular}{|c|c|c|c|}
\hline $\begin{array}{l}\text { Number of } \\
\text { frequency }\end{array}$ & $\begin{array}{l}\text { Shell eigenfrequencies } \\
\text { without electric circuit } \\
\qquad f[\mathrm{~Hz}]\end{array}$ & $\begin{array}{c}\text { Shunt circuit } \\
\text { optimal parameters } \\
\text { for single-mode } \\
\text { damping } \\
\text { L [H], R [kOhm] }\end{array}$ & $\begin{array}{l}\text { Complex eigenfrequencies of the } \\
\text { shell with tuned shunt circuit } \\
\qquad f=f_{\mathrm{Re}}+i f_{\mathrm{Im}}\end{array}$ \\
\hline 1 & 557.41 & $L=6.93, R=5.3$ & $553.60+i 28.30$ \\
\hline 4 & 759.57 & $L=3.66, R=4.1$ & $750.59+i 32.06$ \\
\hline 5 & 803.79 & $L=3.67, R=4.02$ & $813.58+i 31.12$ \\
\hline 12 & 1293.42 & $L=1.3, R=2.18$ & $1308.34+i 48.24$ \\
\hline 15 & 1482.21 & $L=1.04, R=2.57$ & $1493.29+i 81.48$ \\
\hline
\end{tabular}

Table 1: Natural vibration frequencies for the shell with piezoelectric element and no external circuit and with external circuit tuned to a single frequency.

On the basis of the solution to the natural vibration problem for the system (shell with shunted piezoelectric element), there were retrieved relationship surfaces of imaginary parts of complex eigenfrequiencies depending on variation of $R$ and $L$ parameters for the this five frequencies under consideration (Fig. 2)

When analysing relationship surfaces in Fig. 2, one can identify frequencies for which the surfaces intersection exists. These are at the 4th and 5th frequencies (Fig. 3a) and the 12th and 15th frequencies (Fig. 3c).

Let us consider these pairs separately. Figs. $3 \mathrm{~b}$ and $3 \mathrm{~d}$ show the lines of $\omega_{\mathrm{Im}}$ coincidence in the R- $L$ space. Along these lines, damping indices take different values. To define the best damping of structural vibrations for the corresponding pairs, it is necessary to find the values of the external circuit parameters that guarantee the maximal decay rate of vibrations. 


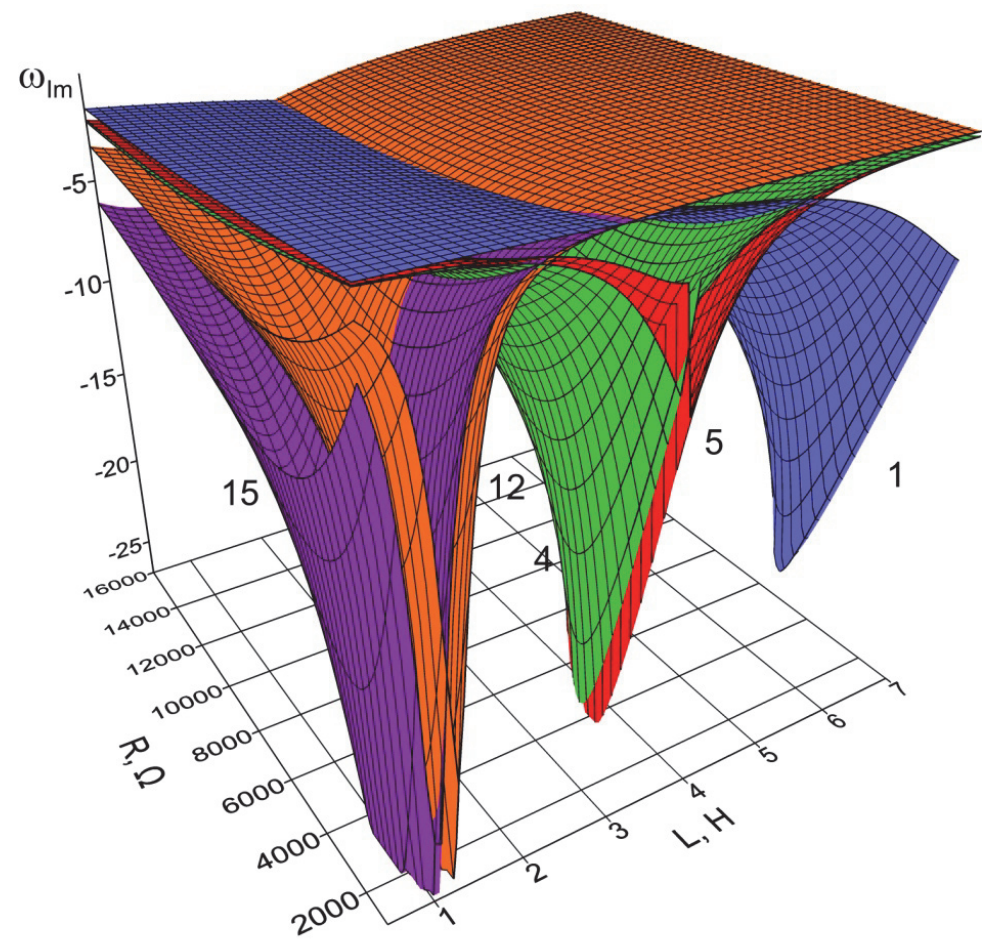

Figure 2: Relationship surfaces of imaginary parts of complex eigenfrequiencies $\omega_{\operatorname{Im}}$ depending on variation of shunt circuit parameters $R$ and $L$ for the five frequencies under study.

It is important to note that although plots of relationship surfaces of damping indices for different complex eigenfrequencies have points and lines of coincidence, numerical results show some difference. This can be explained by the tolerance of setting the optimal parameters of shunting circuit. The more significant digits of $R$ and $L$ values is taken into account the more close to each other the values of imaginary parts are.

\begin{tabular}{|c|c|c|c|c|}
\hline $1^{\text {st }}$ frequency & $4^{\text {th }}$ frequency & $5^{\text {th }}$ frequency & $12^{\text {th }}$ frequency & $15^{\text {th }}$ frequency \\
\hline \multicolumn{5}{|c|}{$\mathrm{R}=4.41[\mathrm{kOhm}], \mathrm{L}=3.57[\mathrm{H}]$} \\
\hline $551.77+i 1.98$ & $755.40+i 23.76$ & $791.50+i 23.58$ & $1296.47+i 0.71$ & $1486.35+i 0.76$ \\
\hline \multicolumn{5}{|c|}{$R=2.45[\mathrm{kOhm}], L=1.19[\mathrm{H}]$} \\
\hline $553.67+i 0.45$ & $754.17+i 1.11$ & $798.66+i 0.93$ & $1281.48+i 25.43$ & $1507.57+i 25.76$ \\
\hline
\end{tabular}

Table 2: Values of complex natural vibration frequencies in the case of parameters of the external circuit that provide maximal and close to each other values of imaginary parts.

Tab. 2 shows the $R$ and $L$ values at which pairs of 4th and 5 th frequencies and 12 th and 15 th frequencies have maximal and almost coincident values of imaginary parts. It also shows the corresponding complex natural vibration frequencies for the five modes under study. The frequencies having maximal imaginary parts, that are very close to each other in magnitude, are highlighted in bold.

A comparison of the imaginary parts presented in Tabs. 1 and 2 allows us to make a conclusion about the possibility of damping two frequencies using one single piezoelectric element shunted with one single series resonant $R L$ circuit with its parameters selected in the appropriate way.

At the same time, the damping indices $\omega_{\mathrm{Im}}$ for 4 th $/ 5$ th and 12 th $/ 15$ th frequencies are lower than those at shunt circuit tuning for single-mode damping, which provides the maximal the decay rate of vibrations for one single mode (see Tab. 
1). However, when compared to other frequencies (see Tab. 2), the damping indices for these pairs of modes appear to be sufficiently higher.

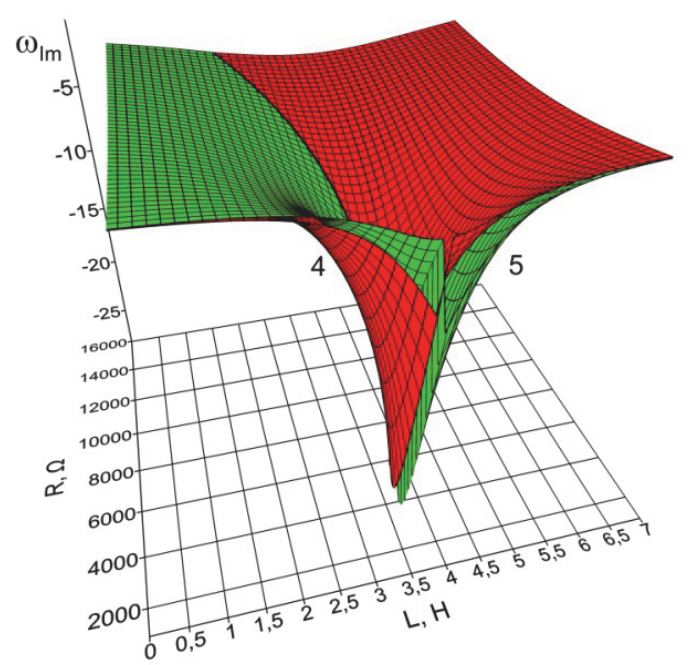

a

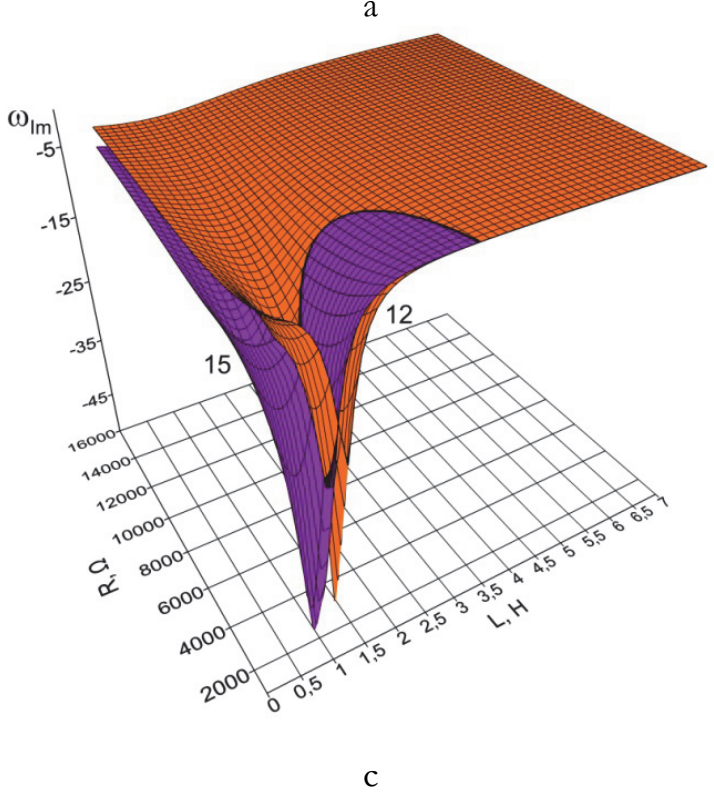

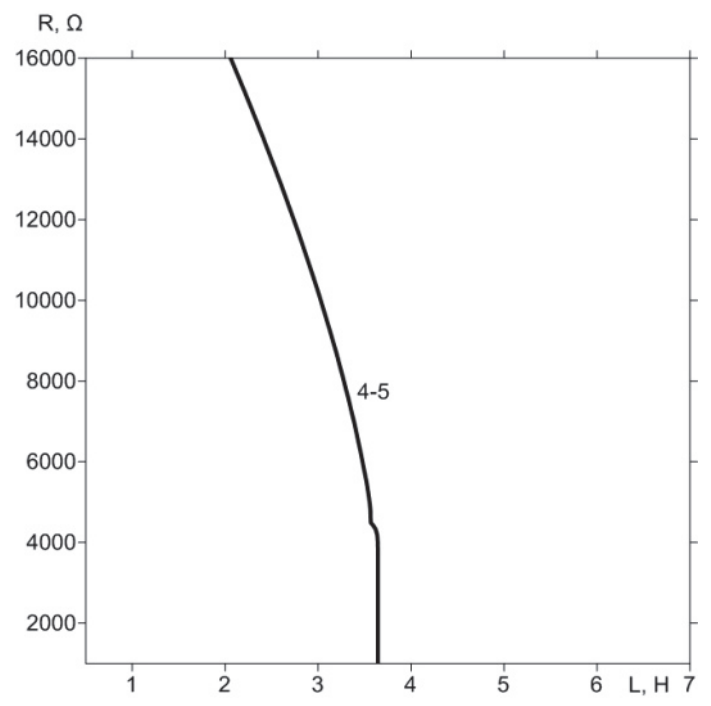

$\mathrm{b}$

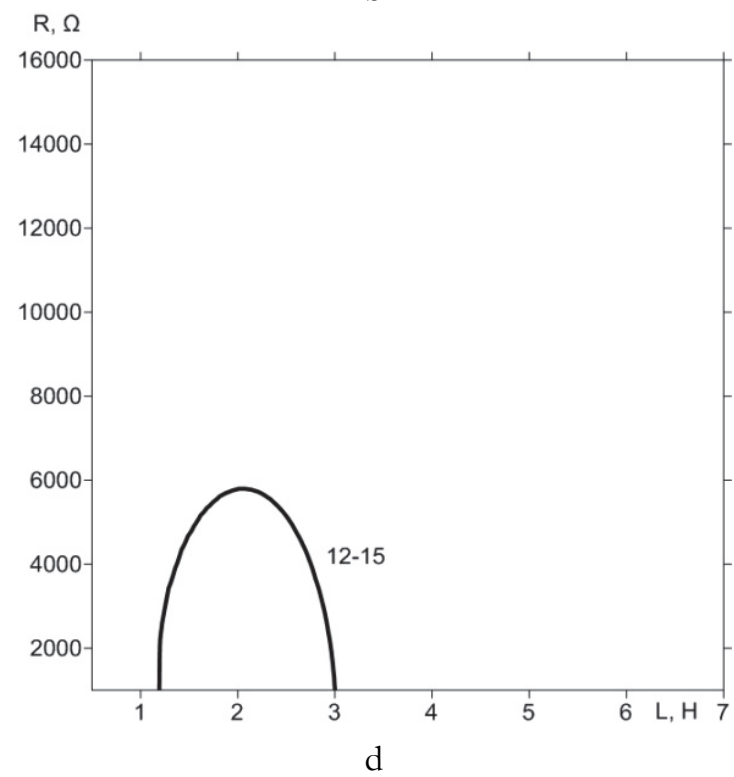

Figure 3: Relationship surfaces of damping indices depending on $R$ and $L$ parameters and projections of the lines of their coincidence for 4 th and 5 th frequencies $(a, b)$ and 12 th and 15 th frequencies $(c, d)$.

Continuing the analysis of relationship surfaces presented in Fig. 2, one might see that in the R-L parameters space there are points where imaginary parts of complex eigenfrequencies coincide for more than two frequencies. Fig. 4 shows the projections of all possible lines of $\omega_{\text {Im }}$ coincidence for which such points were found.

These points correspond to shunt circuit parameters at which it is possible to damp vibrations on three frequencies. These points are labelled with roman numbers: I -4 th, 5th and 12th modes; II -5 th, 12th and 15th modes; III -1 st, 12 th and 15 th modes; IV -4 th, 5 th and 15 th modes.

According to the results that correspond to the point III on fig. 4 and presented in Tab. 3, the efficiency of damping of vibrations with the aid of one and the same technical devices for 1st, 12th and 15th frequencies is quite low. This is 
because although imaginary parts of these frequencies are close to each other, their magnitudes are very low in comparison with other possible options.

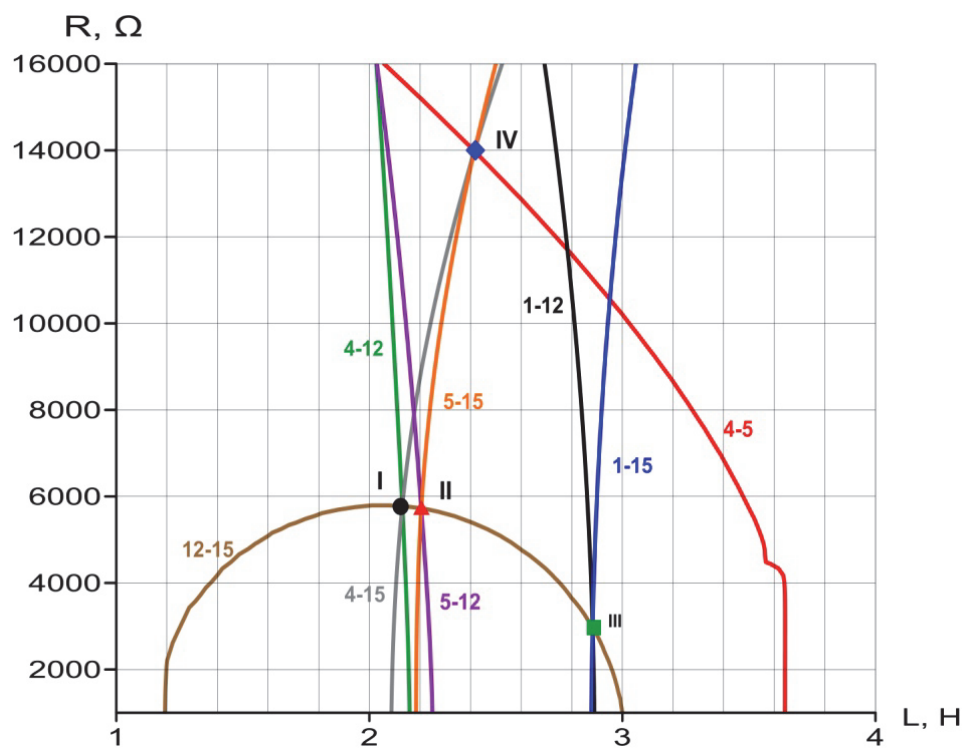

Figure 4: Projections of imaginary parts of complex eigenfrequencies coincidence lines in the R- $L$ parameters space that have points of intersection.

\begin{tabular}{|c|c|c|c|c|c|}
\hline & \multicolumn{5}{|c|}{ The number of eigenfrequence } \\
\hline & 1 & 4 & 5 & 12 & 15 \\
\hline \multirow{2}{*}{ I } & \multicolumn{5}{|c|}{$\mathrm{R}=5.77[\mathrm{kOhm}], \mathrm{L}=2.14[\mathrm{H}]$} \\
\hline & $553.34+i 1.36$ & $753.82+i 4.41$ & $797.98+i 3.75$ & $1298.61+i 4.12$ & $1489.72+i 4.06$ \\
\hline \multirow{2}{*}{ II } & \multicolumn{5}{|c|}{$\mathrm{R}=5.72[\mathrm{kOhm}], L=2.21[\mathrm{H}]$} \\
\hline & $553.28+i 1.38$ & $753.70+i 4.65$ & $797.84+i 3.95$ & $1298.54+i 3.73$ & $1489.49+i 3.68$ \\
\hline \multirow{2}{*}{ III } & \multicolumn{5}{|c|}{$\mathrm{R}=2.94[\mathrm{kOhm}], L=2.88[\mathrm{H}]$} \\
\hline & $552.30+i 1.01$ & $747.78+i 7.20$ & $792.75+i 5.17$ & $1297.78+i 0.96$ & $1487.89+i 0.95$ \\
\hline \multirow{2}{*}{ IV } & \multicolumn{5}{|c|}{$R=13.99[\mathrm{kOhm}], L=2.42[\mathrm{H}]$} \\
\hline & $554.65+i 2.39$ & $757.92+i 4.10$ & $801.76+i 4.09$ & $1295.43+i 2.10$ & $1485.87+i 3.90$ \\
\hline $\mathrm{V}$ & \multicolumn{5}{|c|}{$\mathrm{R}=5.75[\mathrm{kOhm}], L=2.20[\mathrm{H}]$} \\
\hline & $553.30+i 1.39$ & $753.73+i 4.64$ & $797.86+i 3.94$ & $1298.53+i 3.76$ & $1489.49+i 3.71$ \\
\hline
\end{tabular}

Table 3: Parameters $R$ and $L$ of electric circuit at which imaginary parts are very close to each other for more than two frequencies and their corresponding complex eigenfrequencie.

The last row of Tab. 3 (for the point V, see fig.4) gives the complex frequency values that correspond to the circuit parameters selected from the interval constrained by $R$ and $L$ values obtained for groups I and II (points I and II, see fig.4). The results presented in this row show considerably high and comparable values of imaginary parts for the frequencies from the groups mentioned above. This allows us to conclude that appreciable damping of vibrations may be possible at four frequencies (4th, 5th, 12th, 15th) with the aid of one single piezoelectric element shunted with one single series resonant $R L$ circuit if the circuit element parameters are $R=5.75 \mathrm{kOhm}$ и $L=2.20 \mathrm{H}$. 
To illustrate that the possibility of damping properties of a structure with piezoelectric element increases while using a single resonant circuit, Fig. 5 shows the frequency response amplitudes of displacement vector component $U_{r}$ at point $\mathrm{A}$ when the system is excited by harmonic force $P_{r}$ applied at point B (Fig. 1b). The results shown were obtained for shunt circuit tuning for single-mode damping at 4th, 5th, 12th and 15th frequencies and for shunt circuit tuning according to the last row of Tab. 3 that provides damping properties increase for all four frequencies (4th, 5th, 12th, 15th).

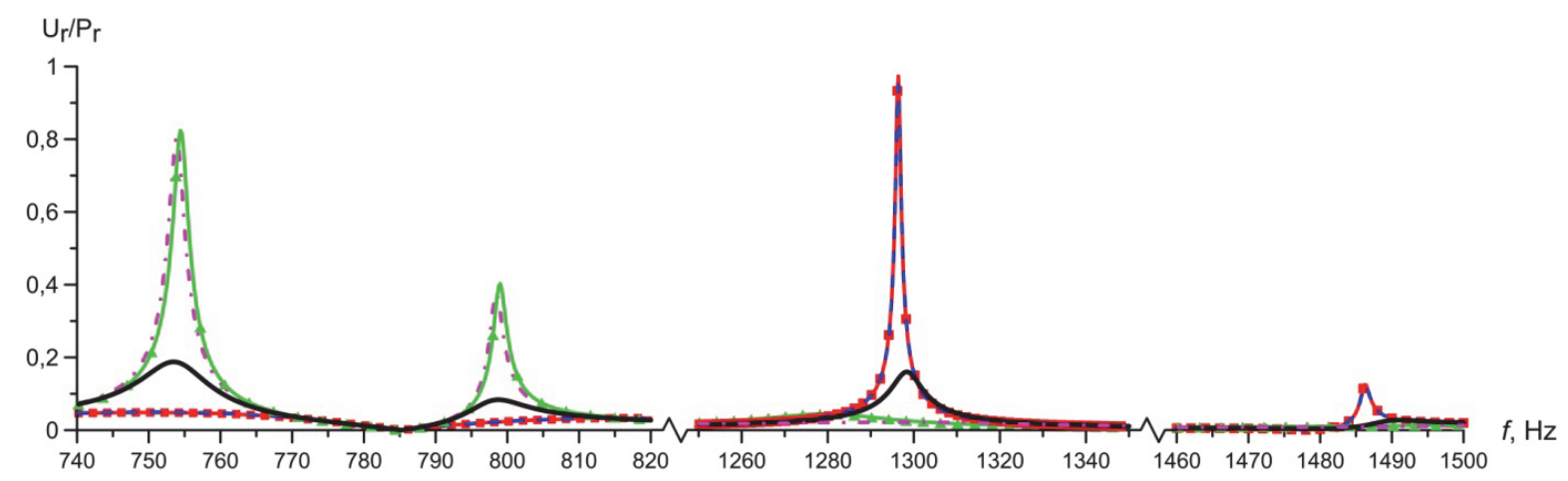

Figure 5: Frequency response amplitudes for the displacement vector component $U_{r}$ at point $\mathrm{A}$ of the shell (Fig. $\left.1 \mathrm{~b}\right)$ at shunt circuit tuning for maximal damping 4th (blue line), 5th (red line), 12th (green line) and 15th (violet line) modes separately and at shunt circuit tuning for multimodal damping of all these (4th, 5th, 12th, 15th) modes (black line).

\section{DEFINITION OF SHUNT CIRCUIT PARAMETERS FOR DAMPING STRUCTURAL VIBRATIONS AT ALL THE FREQUENCIES IN THE SPECIFIED FREQUENCY RANGE}

$\mathrm{T}$ he previous section demonstrated the possibility of damping vibrations for an electromechanical system at several separate frequencies within the specified frequency range using the same parameters as for shunt circuit elements. However, in technical applications it is conventionally necessary to ensure the damping of structural vibrations at all the frequencies within the specified frequency range. Within the framework of the problem under study, this can be achieved if the particular location of the piezoelectric element can be found at which electric potential takes eligible values for its reliant operation at all of the frequencies within the specified frequency range. The searching procedure of optimal location for piezoelectric element that provides possibility of multimodal vibration damping within specified frequency range was presented in [37].

\begin{tabular}{cccc}
\hline $\begin{array}{c}\text { Number of } \\
\text { frequency }\end{array}$ & $\begin{array}{c}\text { Shell } \\
\text { eigenfrequencies } \\
\text { without electric } \\
\text { circuit } f[\mathrm{~Hz}]\end{array}$ & $\begin{array}{c}\text { Shunt circuit optimal } \\
\text { parameters for single-mode } \\
\text { damping R }[\mathrm{kOhm}], \mathrm{L}[\mathrm{H}],\end{array}$ & $\begin{array}{c}\text { Complex eigenfrequencies } \\
\text { of the shell with tuned } \\
\text { shunt circuit } \\
f=f_{\mathrm{Re}}+i_{\mathrm{Im}}\end{array}$ \\
\hline 1 & 557.41 & $R=5434.43, L=6.57$ & $554.26+i 21.36$ \\
2 & 587.68 & $R=5204.20, L=6.32$ & $586.81+i 17.60$ \\
3 & 620.20 & $R=5954.96, L=5.69$ & $636.32+i 33.12$ \\
\hline
\end{tabular}

Table 4: Natural vibration frequencies for the shell with piezoelectric element and no electric circuit and with electric circuit tuned to a single frequency.

The object of the study remains the same: the shell presented in Fig. 1. The goal of the simulations is to provide damping of all the frequencies within the range 500-700 Hz while using the same technical devices: a single piezoelectric element and a single resonant series $R L$ circuit. In this case, the piezoelectric element must be placed so that its centre of mass is offset from the clamped edges at $150 \mathrm{~mm}$ and is at a $30.2^{\circ}$ angle from the free-supported generatrix. Then in the frequency range 500-700 $\mathrm{Hz}$, there will be three natural vibration frequencies at which the piezoelectric element generates sufficient electric potential. By analogy with previous calculations, Tab. 4 shows the natural vibration frequencies of the 
shell with an attached piezoelectric element without an electric circuit, the optimal parameters of the shunt circuit to damp vibrations at a single frequency and the corresponding values of complex eigenfrequencies for the shell with a shunted piezoelectric element.

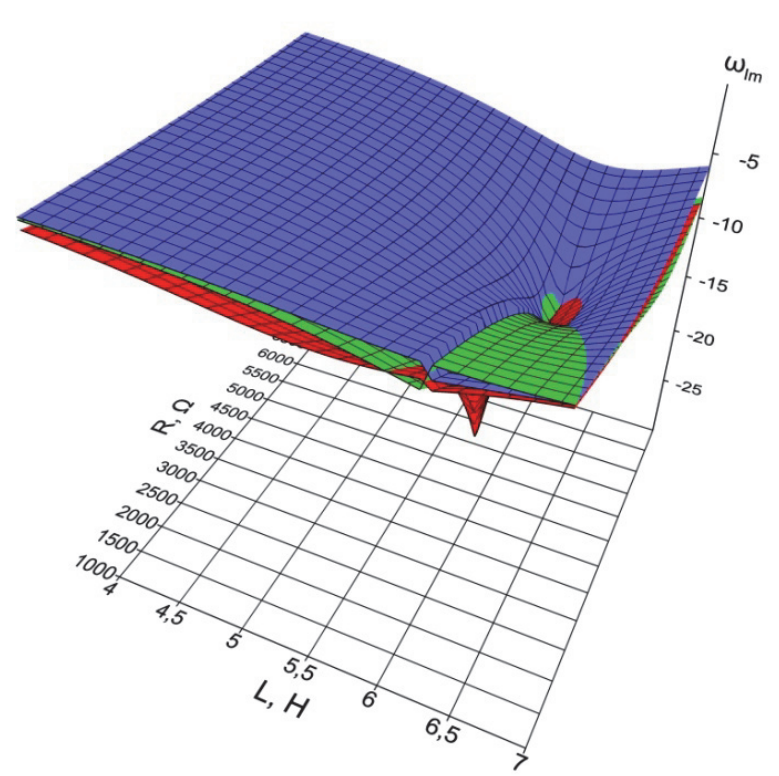

a

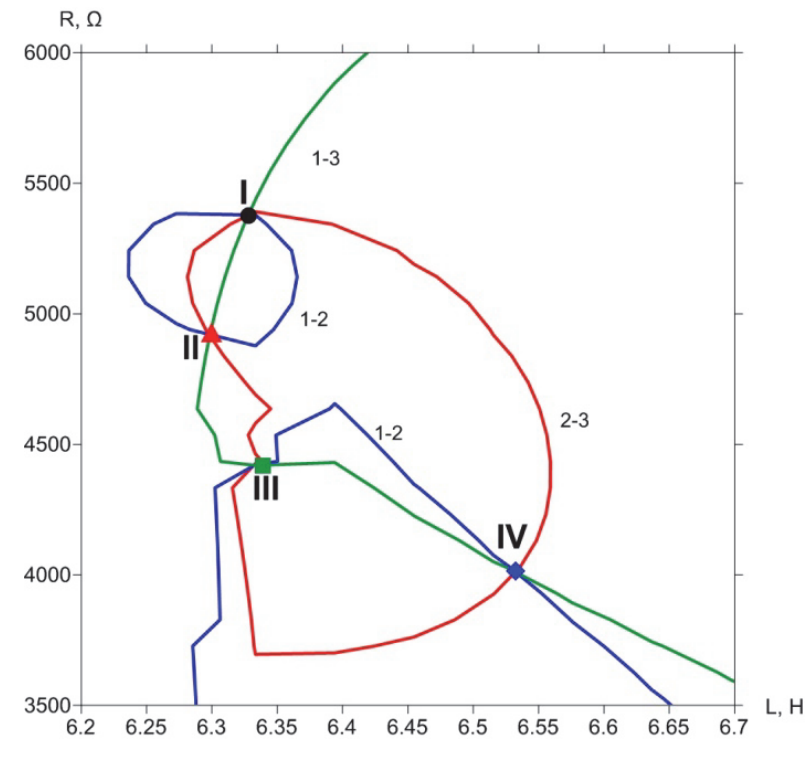

b

Figure 6: Damping indices relationship surfaces depending on $R$ and $L$ parameters (a) and projections of the lines of their pairwise coincidence (b) for the frequencies under consideration.

On the basis of the solution to the natural vibration problem for the shell with shunted piezoelectric element, we retrieved relationship surfaces of damping indices depending on variation of $R$ and $L$ parameters for these three frequencies (Fig. 6a). These relations allow us to define in the R-L space the lines of pairwise coincidence of the imaginary part of the frequencies under consideration. Fig. 6b shows the presented projections of these lines. According to the plots in Fig. 6b, there are points where the damping ratios for all three frequencies appear to coincide. Tab. 5 represents the values of the electric circuit parameters and corresponding values of comlex eigenfrequencies. The results show that option II has the highest values of damping indices.

\begin{tabular}{cccc}
\hline I & & $\mathrm{R}=5.38[\mathrm{kOhm}], L=6.33[\mathrm{H}]$ & \\
& $549.84+i 14.59$ & $586.14+i 13.08$ & $647.18+i 13.36$ \\
II & & $\mathrm{R}=4.92[\mathrm{kOhm}], L=6.30[\mathrm{H}]$ & \\
& $547.17+i 14.77$ & $575.98+i 15.71$ & $648.74+i 13.44$ \\
III & & $\mathrm{R}=4.42[\mathrm{kOhm}], L=6.33[\mathrm{H}]$ & \\
& $573.07+i 12.36$ & $593.87+i 13.86$ & $650.18+i 12.45$ \\
IV & & $\mathrm{R}=4.01[\mathrm{kOhm}], L=6.53[\mathrm{H}]$ & \\
& $569.05+i 10.23$ & $593.96+i 9.73$ & $650.05+i 9.67$ \\
\hline
\end{tabular}

Table 5: Values of $R$ and $L$ at which imaginary parts of the first three complex eigenfrequencies within the frequency range up to 700 $\mathrm{Hz}$ are close to each other.

Fig. 7 shows the frequency response amplitudes of displacement vector component $U_{r}$ at point A when the system is excited by harmonic force $P_{r}$ applied at point $\mathrm{B}$. The results correspond to shunt circuit tuning for single-mode damping of the frequencies under study and to damp all the three modes within the frequency range up to $700 \mathrm{~Hz}$. 
These results show that sometimes, as in this case, shunt circuit parameters corresponding to multimodal damping may provide results comparable to single-mode damping by the amplitude magnitudes.

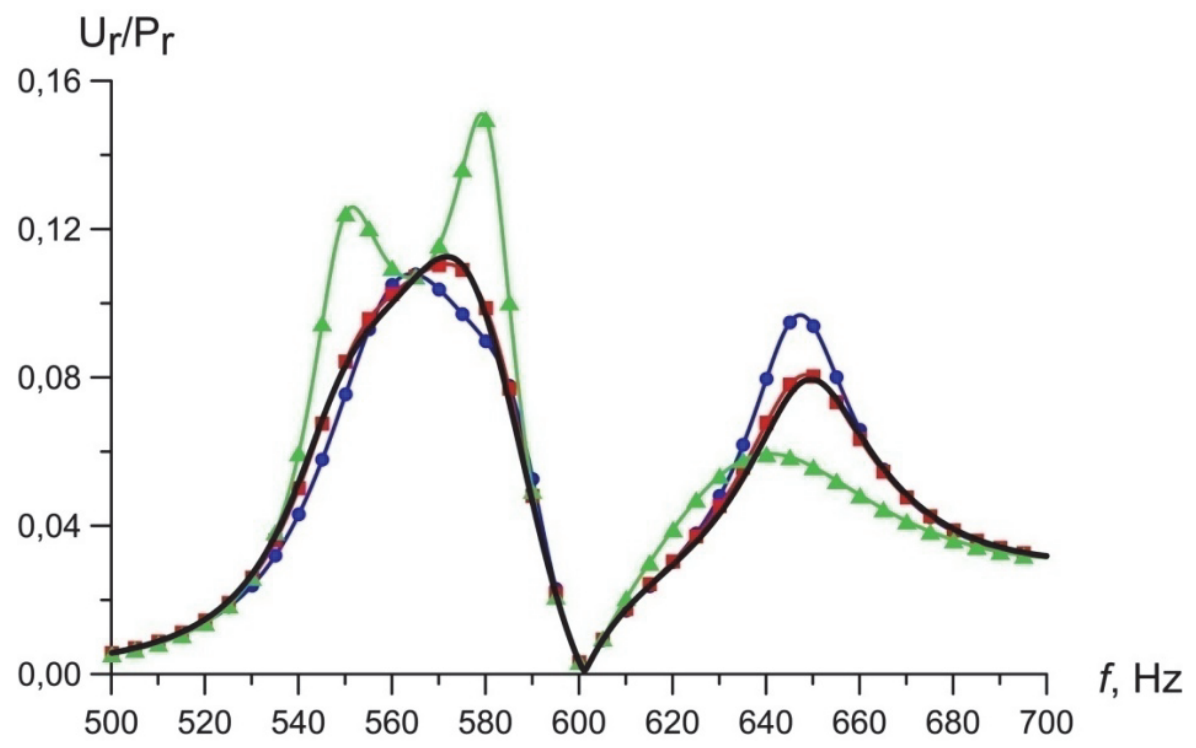

Figure 7: Frequency response amplitudes for point A of the shell (Fig. 1b) at shunt circuit tuning to damp all three (1st, 2nd, 3rd) vibration modes (black line) and to damp each mode separately (1st - blue line, 2nd - red line, 3rd - green line).

\section{CONCLUSIONS}

I $\mathrm{n}$ this paper, we considered the application of a natural vibration problem for piecewise homogeneous bodies with piezoelectric elements and external electric circuits in order to find variants that provide dissipative properties increasing at several vibration modes (multimodal vibration damping) with the aid of a simple shunt circuit, consisting of resistive and inductive elements connected in series. The search for variants that provide multimodal damping is based on the analysis of the behaviour of imaginary parts of complex natural vibration frequencies that define the vibration damping rate for the corresponding frequencies in the space of external electric circuit parameters (resistance and inductance)

It was shown that at a specific piezoelectric element location there are groups of vibration modes that have common lines and points where the imaginary parts of corresponding complex eigenfrequencies coincide in the space of electric circuit parameters (resistance and inductance). The existence of such points allows us to find the shunt circuit tuning options that ensure an increase of the dissipative characteristics of the system at two or more vibration modes.

The options of multimodal vibration damping found in this study were illustrated by frequency response plots at steadystate vibration modes.

\section{ACKNOWLEDGMENTS}

he work is supported by the RFBR (projects No 18-31-00080-mol_a).

\section{REFERENCES}

[1] Forward, R.L. (1979). Electronic damping of vibrations in optical structures, Journal of Applied Optics 18(5), pp. 690697. DOI: $10.1364 /$ AO.18.000690. 
[2] Hagood, N.W., Von Flotow, A. (1991). Damping of structural vibrations with piezoelectric materials and passive electrical networks, Journal of Sound and Vibration 146, pp.243-268. DOI: 10.1016/0022-460X(91)90762-9.

[3] Moheimani, S.O.R., Fleming, A.J. (2006). Piezoelectric transducers for vibration control and damping, Springer Science \& Business Media. DOI: 10.1007/1-84628-332-9.

[4] Viana, F.A.C., Valder, S.Jr. (2006). Multimodal Vibration Damping through Piezoelectric Patches and Optimal Resonant Shunt Circuits, Journal of the Brazilian Society of Mechanical Sciences and Engineering, XXVIII(3), pp.293310. DOI: $10.1590 /$ S1678-58782006000300007.

[5] Cheng, T.H., Oh, I.K. (2009). A current-flowing electromagnetic shunt damper for multi-mode vibration control of cantilever beams, Smart Materials and Structures, 18(9), art. 095036. DOI: 10.1088/0964-1726/18/9/095036.

[6] Behrens, S., Moheimani, S.O.R. (2000). Optimal resistive elements for multiple mode shunt damping of a piezoelectric laminate beam, Proc. of the 39th IEEE Conf. on Decision and Control, 4, pp.4018-4023.

DOI: $10.1109 /$ CDC.2000.912343.

[7] Fleming, A.J., Moheimani, S.O.R. (2003). Adaptive piezoelectric shunt damping, Smart Materials and Structures, 12(1), pp.36-48. DOI: 10.1088/0964-1726/12/1/305.

[8] Behrens S, Moheimani, S.O.R. and Fleming, A.J. (2003). Multiple mode current flowing passive piezoelectric shunt controller, Journal of Sound and Vibration, 266(5), pp.929-942. DOI: 10.1016/S0022-460X(02)01380-9.

[9] Behrens, S. and Moheimani, S.O.R. (2002). Current flowing multiple mode piezoelectric shunt dampener, SPIE Proceedings Series, 4697(24), pp.217-226. DOI: 10.1117/12.472658.

[10] Wu, S.Y. and Bicos, A.S. (1997). Structural vibration damping experiments using improved piezoelectric shunts, SPIE Proceedings Series, 3045, pp. 40-50. DOI: 10.1117/12.274217.

[11] Wu, S.Y. (1999). Multiple PZT transducers implemented with multiple-mode piezoelectric shunting for passive vibration damping, SPIE Proceedings Series, 672, pp. 112-122. DOI: 10.1117/12.349774.

[12] Wu, S.Y. (1998). Method for multiple mode shunt damping of structural vibration using a single PZT transducer, SPIE Proceedings Series, 3327, pp.159-168. DOI: 10.1117/12.310680.

[13] Hollkamp, J.J. (1994). Multimodal passive vibration suppression with piezoelectric materials and resonant shunts, Journal of intelligent material systems and structures, 5, pp. 49-56. DOI: 10.1177/1045389X9400500106.

[14] Yan, L., Lallart, M. and Guyomar, D. (2014). Multimodal nonlinear damping technique using spatial filtering, Journal of Intelligent Material Systems and Structures, 25(3), pp. 308-320. DOI: 10.1177/1045389X13493355.

[15] Goldstein, A. (2011). Self-Tuning Multimodal Piezoelectric Shunt Damping, J. Braz. Soc. Mech. Sci. \& Eng., XXXIII(4), pp. 428-436. DOI: 10.1590/S1678-58782011000400006.

[16] Vidoli, S and dell'Isola, F. (2001). Vibration control in plates by uniformly distributed PZT actuators interconnected via electric networks, European Journal of Mechanics/A Solids, 20, pp. 435-456.

DOI: $10.1016 /$ S0997-7538(01)01144-5.

[17] Porfri, M., dell'Isola, F. and Frattale, M.F.M. (2004). Circuit analog of a beam and its application to multimodal vibration damping, using piezoelectric transducers, International Journal of Circuit Theory and Applications, 32, pp. 167-198. DOI: 10.1002/cta.273.

[18] dell'Isola, F., Henneke, E.G. and Porfiri, M. (2003). Piezo-electromechanical structures: new trends towards the multimodal passive vibration control, SPIE Proceedings Series, 5052, pp. 392-402. DOI: 10.1117/12.483803.

[19] Maurini, C., dell'Isola, F. and Del Vescovo, D. (2004). Comparison of piezoelectronic networks acting as distributed vibration absorbers, Mechanical Systems and Signal Processing, 18(5), pp. 1243-1271.

DOI: $10.1016 /$ S0888-3270(03)00082-7.

[20] Giorgio, I., Culla, A. and Del Vescovo, D. (2009). Multimode vibration control using several piezoelectric transducers shunted with a multiterminal network, Archive of Applied Mechanics, 79(9), pp. 859-879.

DOI: $10.1007 /$ s00419-008-0258-x.

[21] Lossouarn, B., Aucejo, M., and Deü, J.F. (2015). Multimodal coupling of periodic lattices and application to rod vibration damping with a piezoelectric network, Smart Materials and Structures, 24 (4), art. 045018. DOI: $10.1088 / 0964-1726 / 24 / 4 / 045018$.

[22] Lossouarn, B., Aucejo, M., and Deü, J.F. (2015). Multimodal vibration damping through a periodic array of piezoelectric patches connected to a passive network, Smart Materials and Structures, 24 (11), art. 115037. DOI: $10.1088 / 0964-1726 / 24 / 11 / 115037$.

[23] Casadei, F., Ruzzene, M., Dozio, L. and Cunefare, K.A. (2010). Broadband vibration control through periodic arrays of resonant shunts: experimental investigation on plates, Smart Materials and Structures, 19(1), art.015002.

DOI: $10.1088 / 0964-1726 / 19 / 1 / 015002$. 
[24] Guo, K.M. and Jiang, J. (2014). Independent modal resonant shunt for multimode vibration control of a truss-cored sandwich panel, International Journal of Dynamics and Control, 2, pp. 326-334. DOI: 10.1007/s40435-013-0036-7.

[25] Trindade, M.A. and Maio, C.E.B. (2008). Multimodal passive vibration control of sandwich beams with shunted shear piezoelectric materials, Smart Materials and Structures, 17, art. 055015. DOI: 10.1088/0964-1726/17/5/055015.

[26] Spadoni, A., Ruzzene, M. and Cunefare, K. (2009). Vibration and Wave Propagation Control of Plates with Periodic Arrays of Shunted Piezoelectric Patches, Journal of Intelligent Material Systems and Structures, 20(5), pp. 979-990. DOI: $10.1177 / 1045389 X 08100041$.

[27] Casadei, F., Beck, B., Cunefare, A. and Ruzzene, M. (2012). Vibration control of plates through hybrid configurations of periodic piezoelectric shunts, Journal of Intelligent Material Systems and Structures, 23(10), pp. 1169-1177. DOI: $10.1177 / 1045389 X 12443014$.

[28] Berardengo, M., Manzoni, S., Conti, A.M. (2017). Multi-mode passive piezoelectric shunt damping by means of matrix inequalities, Journal of Sound and Vibration, 405(29), pp. 287-305. DOI: 10.1016/j.jsv.2017.06.002.

[29] Kligman, E.P., Matveenko, V.P. (1997). Natural Vibration Problem of Viscoelastic Solids as Applied to Optimization of Dissipative Properties of Constructions, International Journal of Vibration and Control, 3(1), pp. 87-102. DOI: $10.1177 / 107754639700300107$.

[30] Kligman, E.P., Matveenko, V.P., Sevodina, N.V. (2010) Determination of natural vibrations of piecewise homogeneous viscoelastic bodies using the ANSYS software package, Computational Continuum Mechanics, 3(2), pp. 46-54. DOI: 10.7242/1999-6691/2010.3.2.16.

[31] Parton, V.Z. and Kudryavtsev, B.A. (1988). Electro-magnetoelasticity: Piezoelectrics and Electrically Conductive Solids, New York: Gordon and Breach Science Publishers Ltd. ISBN-13: 978-2881246715.

[32] Karnaukhov, V.G. and Kirichok, I.F. (1988). Electrothermal viscoelasticity, Kiev: Nauk. Dumka.

[33] Matveenko, V.P., Oshmarin, D.A., Sevodina, N.V. and Iurlova, N.A. (2016). Natural vibration problem for electroviscoelstic body with external electric circuits and finite-element relations for its numerical implementation, Computational Continuum Mechanics, 9(4), pp. 476-485. DOI: 10.7242/1999-6691/2016.9.4.40.

[34] Iurlova, N., Sevodina, N., Oshmarin, D. and Iurlov, M. (2019). Algorithm for solving problems related to the natural vibrations of electro-viscoelastic structureswith shunt circuits using ANSYS data, International Journal of Smart and Nano Materials, 10(2), pp. 156-176. DOI: 10.1080/19475411.2018.1542356.

[35] Matveenko, V.P., Iurlova, N.A., Oshmarin, D.A., Sevodina, N.V. and Iurlov, M.A. (2018), An approach to determination of shunt circuits parameters for damping vibrations, International Journal of Smart and Nano Materials, 9(2), pp. 135-149. DOI: 10.1080/19475411.2018.1461144.

[36] Sevodina, N.V., Yurlova, N.A. and Oshmarin, D.A. (2015). The optimal placement of the piezoelectric element in a structure based on the solution of the problem of natural vibrations, Solid State Phenomena, 243, pp.67-74. DOI: 10.4028/www.scientific.net/SSP.243.67.

[37] Oshmarin, D.A., Iurlova, N.A., Sevodina, N.V. and Iurlov, M.A. (2019). Algorithm for the layout of a piezoelectric element in an elastic medium providing the maximal piezoelectric effect within a specified frequency range, International Journal of Smart and NanoMaterials, DOI: 10.1080/19475411.2019.1576070. 